\title{
Driver's Eco-Driving Behavior Evaluation Modeling Based on Driving Events
}

\author{
Chen Chen $\left(\mathbb{D},{ }^{1}\right.$ Xiaohua Zhao $\mathbb{D}^{1},{ }^{1}$ Ying Yao, ${ }^{1}$ Yunlong Zhang, \\ Jian Rong, ${ }^{1}$ and Xiaoming Liu ${ }^{1}$ \\ ${ }^{1}$ Beijing Key Laboratory of Traffic Engineering, Beijing Engineering Research Center of Urban Transport Operation Guarantee, \\ Beijing University of Technology, Beijing 100124, China \\ ${ }^{2}$ Texas A\&M University, College Station, TX 77843, USA
}

Correspondence should be addressed to Xiaohua Zhao; zhaoxiaohua@bjut.edu.cn

Received 17 August 2017; Revised 7 December 2017; Accepted 17 December 2017; Published 18 January 2018

Academic Editor: Yue Liu

Copyright (C) 2018 Chen Chen et al. This is an open access article distributed under the Creative Commons Attribution License, which permits unrestricted use, distribution, and reproduction in any medium, provided the original work is properly cited.

\begin{abstract}
Eco-driving is an effective means to reduce vehicle fuel consumption. Although many researches and devices have been developed to introduce eco-driving, quantitative effects of driver behaviors on fuel consumption are still unclear, as well as quantitative ecodriving advices. To solve this problem and promote the application of eco-driving in China, a driving-events-based eco-driving behavior evaluation model was proposed in this paper. First, based on taxicab operating data, the relationship between three vehicle operating parameters (speed, acceleration, and driving mode duration) and fuel consumption was analyzed. Then, nine fuel-consumption-involved driving events (including Accelerating Sharply, Decelerating Sharply, and Long-Time Accelerating) were proposed and defined. Using the frequency of each driving event in a certain distance as independent variable and vehicle fuel consumption as dependent variable, principal component analysis (PCA) and multiple linear regression were applied to establish driver's eco-driving behavior evaluation model. The model was proved to be highly accurate (96.72\%). At last, based on the evaluation model, corresponding quantitative eco-driving advices were provided to help driver to improve their driving skills.
\end{abstract}

\section{Introduction}

Road transportation consumes large amounts of oil resources (about 40\%) in 2015 [1] and contributes nearly $80 \%$ in air pollutant emission [2]. Some technological breakthroughs are potential to solve this problem, for instance, the usage of advanced engine control system and electric powered vehicles. However, due to existing technological challenges and public attitudes, the popularization of such methods is likely to take a long time.

On a short-term horizon, change in driver behavior, namely, the "eco-driving," arises as a promising method to reduce energy use. Eco-driving includes several driving methods that could achieve higher energy efficiency, so as to reduce fuel consumption and exhaust emission. It has been verified that different driving method could result in different fuel consumption and emission. De Vlieger [3] found that fuel consumption and emission increased by $12 \%-40 \%$ and $20 \%-50 \%$, respectively, when driving aggressively. Van Mierlo et al. [4] indicated that a considerable reductions of $5 \% \sim 25 \%$ in fuel consumption can be achieved after fuelsaving driving practice.

According to previous studies, the application of ecodriving can mainly be classified into three categories: route planning, trajectory optimization, and driver behavior improvement.

Route planning mainly deals with the questions on macroscopic level, for example, the traffic congestion avoidance. Pham et al. [5] developed a participatory sensing service FUEOOGLE, which allowed drivers to find the most fuelefficient routes by plotting vehicle fuel consumption on city road network. Barth et al. [6] developed an environmentally friendly navigation algorithm, which took advantage of mobile-source energy and emission models and minimized fuel consumption and pollutant emissions. Further, Boriboonsomsin et al. [7] presented another eco-routing 
navigation system by taking real-time traffic information into consideration. Yao and Song [8] collected emission and fuel consumption data, based on which a set of mesoscopic vehicle emission and fuel consumption model is established. What is more, an eco-route planning algorithm is proposed.

Trajectory optimization focuses on mesoscopic level. By collecting and utilizing road gradients data, traffic signal information, leading car speed, and so on, the most fuelefficiency trajectory is designed. Barth et al. [9] developed a dynamic eco-driving velocity planning algorithm, which used signal phasing and timing information of an arterial corridor. The algorithm then was tested and showed 12\% reduction of fuel consumption and $\mathrm{CO} 2$ emission. Mandava et al. [10] developed arterial velocity planning algorithms that provided dynamic speed advice to drivers by using the signal information when approaching an intersection. It was verified that energy/emission savings were $12 \%-14 \%$ when using velocity planning. Chen [11] developed an optimization model to determine the optimal eco-driving trajectory at signalized intersection using signal phases information. The case study showed that eco-driving could reduce emissions by $50 \%$.

Driver behavior feedback aims at the improvement of individual driver's vehicle maneuvering from microscopic level. By providing real-time/trip-summarized eco-driving advices during/after travels, drivers adjust their driving behavior to achieve a higher fuel economy. Boriboonsomsin et al. [12] introduced an eco-driving feedback device that could provide both real-time and detained trip emission information. The real-time feedback information included fuel consumption and $\mathrm{CO}_{2}$ emission in a color scheme from red (poor) to green (good). The detained trip information displayed total travel time, average speed, total fuel consumption, and $\mathrm{CO}_{2}$ emission. Application effects from 20 samples showed that average fuel economy improved by $6 \%$ in city streets. Frank et al. [13] implemented an android application that gathered relevant data from vehicle's CAN bus, providing a driver with second-by-second representative eco-score. The eco-score is classified into four categories: BAD, AVERAGE, GOOD, and VERY GOOD, derived by three fuzzified variables (velocity, road slope, and power consumption) based on a fuzzy system. By introducing drivers the basic ecodriving concepts and recommendations after travel, a sevenvolunteers-participated experiment showed that the android application significantly reduced overall energy consumption. Araújo et al. [14] presented a smartphone application that could detect driving pattern and then suggested new behaviors in real-time to reduce fuel consumption. Variables used to classify driving pattern included speed, acceleration, road slope, fuel consumption, throttle signal, and engine rotations. Also, the fuzzy system is used in the classification and feedback model. Eight hints such as "shift gear earlier" and "accelerating too high" were provided to drivers during travel. Wu et al. [15] trained 22 recruited drivers based on a driving simulator by coaching them how to take fuel economy manipulations. The monitoring of fuel consumption before and after training showed a $5.45 \%$ reduction. Besides, Wu et al. [16] developed an Internet + based eco-driving support platform with a cellphone as feedback device to provide fuel consumption and behavior deficiency to drivers after travel. A validation of 50 taxicab drivers' fuel consumption during a month decreases by $4.5 \% \sim 13 \%$.

Among the three eco-driving application categories, route planning and trajectory optimization highly rely on the integration of detailed traffic information and road alignment data. These methods may play tremendous roles under connected vehicle or automatic vehicle circumstance. At present, the developing foundation of connected vehicle and automatic vehicle in China limits their application. Conversely, driver behavior feedback is not subjected to these limitations and has huge room for improvement. Thus, the improvement of driver behavior is paid more attention.

Generally, the keys of improving driver eco-driving behavior are (1) evaluation of drivers' eco-driving behavior and (2) quantitative and operational driving advices feedback. The former is to inform drivers why they are fuelguzzling by identifying drivers' eco-driving behavior deficiency and the latter is to help drivers to improve driving skills.

As to the first aspect (evaluation of drivers' eco-driving behavior), the detection of high-fuel-consumption behavior use in applications mentioned above is mostly achieved by monitoring abnormal fuel usage and consequently provides warning (red/green colors, voices, etc.) messages through on-board screen or smartphone. Some developers have proposed several behavior-based identification algorithms, for example, the fuzzy-system-based eco-score assessment. Nevertheless, the thresholds used in their models mainly derive from experience judgment, other than quantitative analysis based on driving behavior. Also, in the research field of driver behavior evaluation, there are some researches concentrating on the effect of driving styles on fuel consumption. Ferreira et al. [17] studied the impact of driving styles on fuel consumption, exploiting parameters including speed, acceleration, engine rotation speed, and so on, which were collected from vehicle CAN bus and GPS devices. Naive Bayes' algorithm was used to identify the main influence factors that had major effects on fuel consumption. Eleven events like excessive acceleration were classified into five classes (namely, extreme below average, below average, on average, above average, and extreme above average), according to the frequency of events per $100 \mathrm{~km}$. Then, probabilities for fuel consumption of each class were provided. However, this study indicated the probability that how driver behavior impacts fuel consumption, while the accurate quantitative relationship between driving behavior and fuel consumption was still unclear. Other studies [3, 18, 19] mainly focused on the influence of different driving styles classes (aggressive, normal, moderate, conservative, etc.) on energy usage, which was too abstract in practical application.

As to the second aspect (driving advices feedback), most of eco-driving advices which are provided to driver are qualitative at present, for example, "Avoid sharp acceleration." Drivers need to adjust their manipulation cyclically (a process like "driving, feedback, driving with low acceleration, feedback, driving with lower acceleration. ..") to approach a better fuel economy. What is more, it seems that process-based ecodriving advice may be easier to understand and operate by 
drivers than instantaneous-based advice. For example, the message "Accelerate $X X \mathrm{~km} / \mathrm{h}$ within $Y \mathrm{~s}$ " may be better than "Acceleration should be less than $X X \mathrm{~m} / \mathrm{s}^{2}$." Therefore, the concept, driving events, which are defined as typical driving action during a period of time such as accelerating and decelerating, is proposed and used in this paper.

In fact, eco-driving deficiency detection and corresponding advices are integrated. When one knows where and why he/she makes mistake, he/she can correct it quickly. Therefore, the objectives of this paper are as follows:

(1) To quantitatively explore how driver behaviors (driving events) affect fuel consumption, thus evaluating driver's eco-driving behavior.

(2) To propose more quantitative and clearer driving advices on drivers' operating behaviors, thus guiding drivers to improve fuel economy.

In summary, to promote the spreading and application of eco-driving in China, this paper is proposed to establish a driving-event-based eco-driving behavior evaluation model and provide quantitative eco-driving advices. The detection of these driving events and the development of the model can help drivers to realize their driving behavior deficiency and then correct it.

The remainder of the paper is organized as follows. Section 2 introduces the data collection and processing. Then, in Section 3, the proposing of driving events is presented. In Section 4 the establishment of the model is built and ecodriving advices are provided. What is more, in Section 5 the method and results of this paper are discussed and directions of future work are provided. Finally, in Section 6 the conclusions of this paper are summarized.

\section{Data Collection and Processing}

2.1. Vehicle Fuel Consumption and Operating Data Collection. In this paper, 307 taxicabs' fuel consumption and operating data in one day were collected second by second through Beidou/Global Positioning System (GPS) dual-mode positioning device and on-board diagnostic (OBD) device mounted on vehicles. Collected data included time, vehicle instantaneous fuel consumption $(\mathrm{L} / \mathrm{h})$, speed $(\mathrm{km} / \mathrm{h})$, engine speed (rounds per minute, RPM), engine torque $(\mathrm{N} \cdot \mathrm{m})$, cumulative travel distance $(\mathrm{km})$, and vehicle's location information (i.e., the latitude and longitude). All taxicabs used in the study were Hyundai Elantra, with 4-cylinder 1.6 liter engine, and their fuel consumption was certified by the National Level IV emission standard. All of these cabs were put into operation in 2013 and were regularly maintained every $5000 \mathrm{~km}$ complied with taxicab company requirement. So it was assumed that all of taxicabs had almost the same working condition and fuel consumption characteristics. The amount of the collected data was 1.14 million, and the total driving time was 3176 hours. On average, every driver's daily driving time was about 10.3 hours.

2.2. Data Screening and Processing. In this paper, the evaluation of driver's behavior under normal condition is mainly concerned. Thus, data in following cases are not taken into consideration.
(1) Speed $>100 \mathrm{~km} / \mathrm{h}$. Taxicabs used in this paper are mainly operated on Beijing urban road, with the highest speed limit of $80 \mathrm{~km} / \mathrm{h}$. Due to the strict management of taxicab company, speeding is rare. Therefore, data with vehicle speed more than $100 \mathrm{~km} / \mathrm{h}$ (illegal drive) are ignored.

(2) Absolute Value of Acceleration $>20 \mathrm{~km} / \mathrm{h} / \mathrm{s}$. Acceleration can be calculated through vehicle speed. Data with vehicle acceleration more than $20 \mathrm{~km} / \mathrm{h} / \mathrm{s}$ or lower than $-20 \mathrm{~km} / \mathrm{h} / \mathrm{s}$ is judged beyond engine's physical capabilities [20, 21].

Besides, data with values missing more than consecutive 2 seconds in time sequence are labeled. In the following analysis, data above and below the labeled data item are regarded as two different driving processes.

Figure 1 plots the distributions of daily speed and fuel consumption per hundred kilometers (FPH, L/100 km, calculated as (1)) of all the 307 taxicabs in the data-collected day. The results show that taxis' average speed mainly distributes in $20-30 \mathrm{~km} / \mathrm{h}$ in one day. FPHs are generally in the range of $7-10 \mathrm{~L} / 100 \mathrm{~km}$ with a nearly normal distribution.

$\mathrm{FPH}(\mathrm{L} / 100 \mathrm{~km})$

$$
\begin{aligned}
= & \frac{\sum(\text { instantaneous fuel consumption }(\mathrm{L} / \mathrm{h}) / 3600)}{\sum(\text { speed }(\mathrm{km} / \mathrm{h}) / 3600)} \\
& \times 100,
\end{aligned}
$$

where $\mathrm{FPH}$ is $\mathrm{FPH}$ of every driver in the data-collected day.

\section{Defining Driving Events}

3.1. Influence of Vehicle Operating Parameters on Fuel Consumption. Drivers' different operating behaviors result in various vehicle operating states. In this paper, several operating parameters including speed $(\mathrm{km} / \mathrm{h})$, acceleration $(\mathrm{km} / \mathrm{h} / \mathrm{s})$, and driving mode duration (s) are used to evaluate driver's eco-driving behavior. Speed and acceleration have been widely proved to have significant influence on vehicle fuel consumption [22], while few studies concern about driving mode duration. Driving mode duration refers to the duration time of one driving mode. For example, if drivers maintain accelerating for 3 seconds, the driving mode duration of this process is 3 . The reason why driving mode duration is taken into consideration is the positive correlation between vehicle instantaneous fuel consumption (IFC, L/h) and the duration time of acceleration when preprocessing the data, as shown in Figure 2. When continuously accelerating, IFC grows continuously. However, when acceleration process is interrupted by cruising or deceleration, IFC would decrease and IFC in the beginning of next accelerating process is lower than that when continuously accelerating.

The influence of three parameters on vehicle fuel consumption is shown in Figure 3. FPH (its calculation method is similar to (1), while for each speed value, only the samples that have the same speed are used, which can represent the average FPH under each speed case) is used as the measurement for speed, and IFC (for each acceleration value, average IFC is used) is chosen as the measurement of acceleration and driving mode duration. 


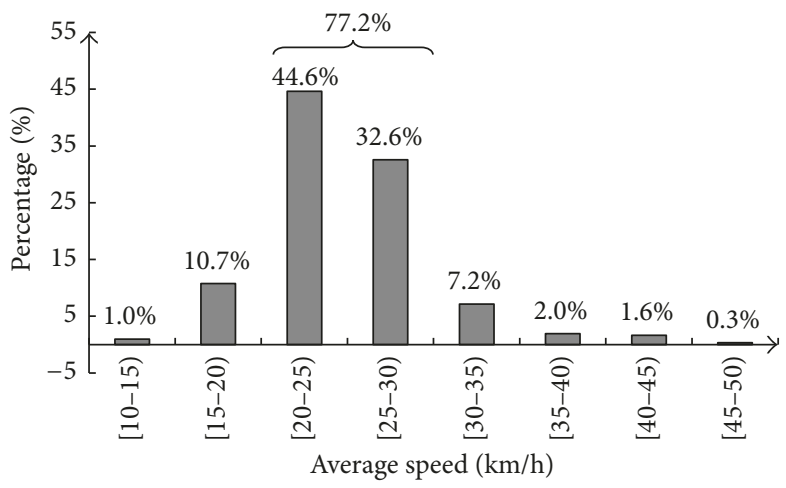

(a) Average speed

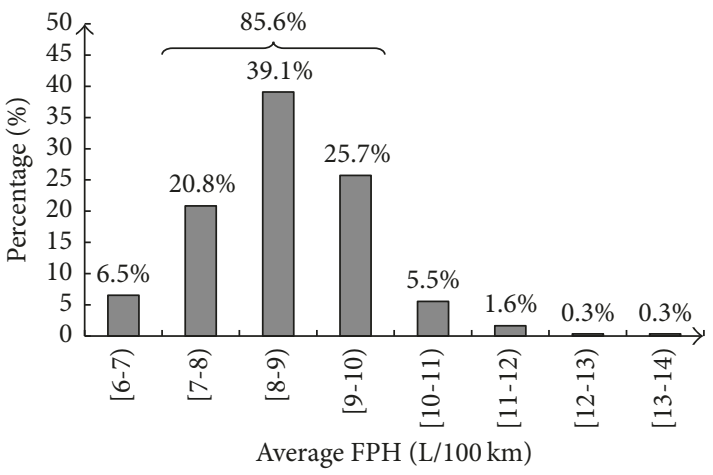

(b) Average FPH

FIGURE 1: Distributions of average daily speed and FPH.

\begin{tabular}{|c|c|c|c|}
\hline Time (yyyymmddhhmmss) & Vehicle ID & Speed $(\mathrm{km} / \mathrm{h})$ & Instantaneous fuel consumption $(\mathrm{L} / \mathrm{h})$ \\
\hline 20140815124154 & $11 \mathrm{BP} 8350$ & 0 & $\square 0.903221$ \\
\hline 20140815124155 & 11BP8350 & 0 & $\square 0.866404$ \\
\hline 20140815124156 & 11BP8350 & 0 & $\square 1.157593$ \\
\hline 20140815124157 & 11BP8350 & 1 & 5.73186 \\
\hline 20140815124158 & $11 \mathrm{BP} 8350$ & 10 & 5.15094 \\
\hline 20140815124159 & 11BP8350 & 22 & 5.79366 \\
\hline 20140815124200 & 11BP8350 & 27 & 6.60942 \\
\hline 20140815124201 & 11BP8350 & 29 & 7.46226 \\
\hline 20140815124202 & 11BP8350 & 36 & 7.80216 \\
\hline 20140815124203 & 11BP8350 & 38 & 7.8516 \\
\hline 20140815124204 & 11BP8350 & 40 & 2.475 \\
\hline 20140815124205 & $11 \mathrm{BP} 8350$ & 40 & 2.078204 \\
\hline 20140815124206 & 11BP8350 & 41 & 5.12004 \\
\hline 20140815124207 & 11BP8350 & 42 & 5.24982 \\
\hline 20140815124208 & 11BP8350 & 44 & 5.5341 \\
\hline 20140815124209 & 11BP8350 & 48 & 6.36222 \\
\hline 20140815124210 & $11 \mathrm{BP} 8350$ & 50 & 6.26334 \\
\hline 20140815124211 & 11BP8350 & 51 & 2.91996 \\
\hline 20140815124212 & 11BP8350 & 50 & 0.961562 \\
\hline 20140815124213 & 11BP8350 & 50 & 1.198728 \\
\hline 20140815124214 & 11BP8350 & 49 & 1.908836 \\
\hline 20140815124215 & $11 \mathrm{BP} 8350$ & 50 & 4.61328 \\
\hline 20140815124216 & 11BP8350 & 50 & 3.202268 \\
\hline 20140815124217 & 11BP8350 & 51 & 4.84812 \\
\hline 20140815124218 & 11BP8350 & 52 & 5.50938 \\
\hline
\end{tabular}

Raw data

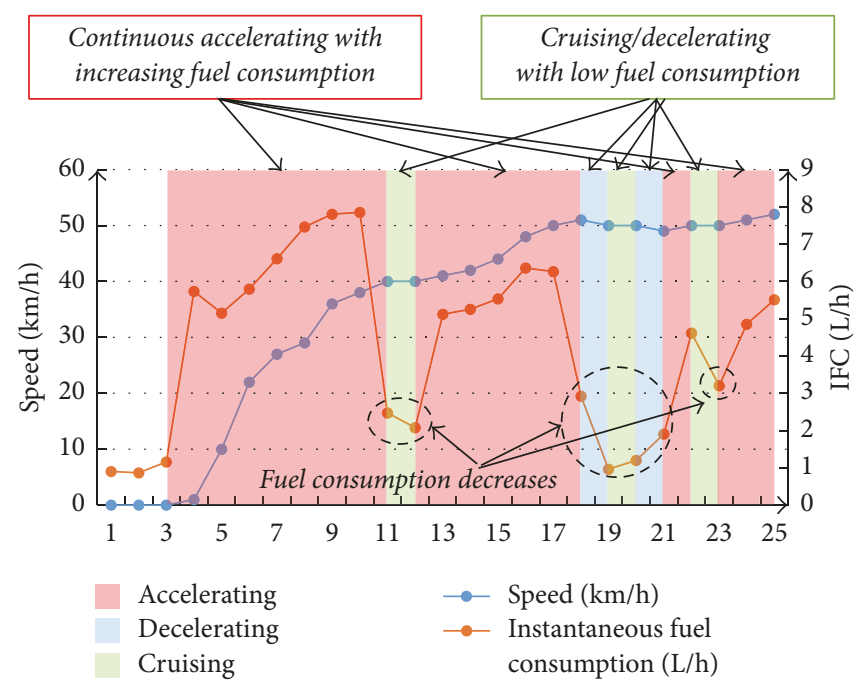

Line chart of raw data

FIgURE 2: Positive correlation between vehicle instantaneous fuel consumption and the duration time of acceleration. 


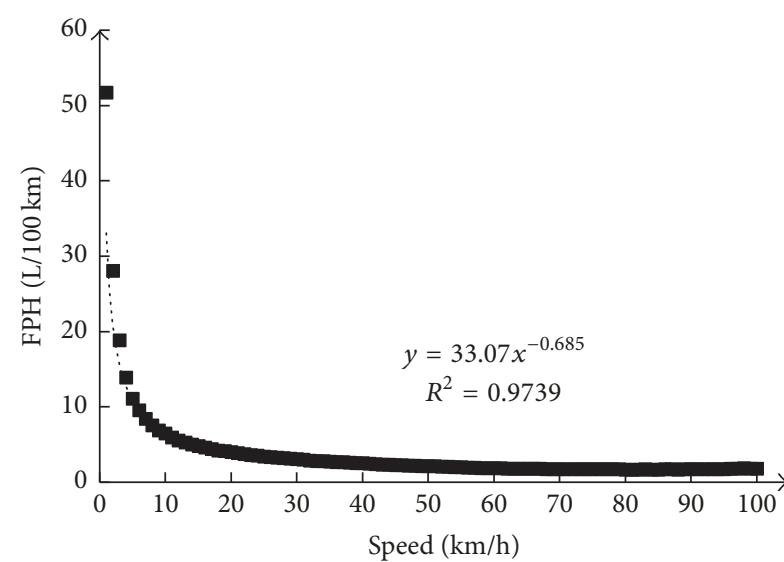

(a) Speed-FPH

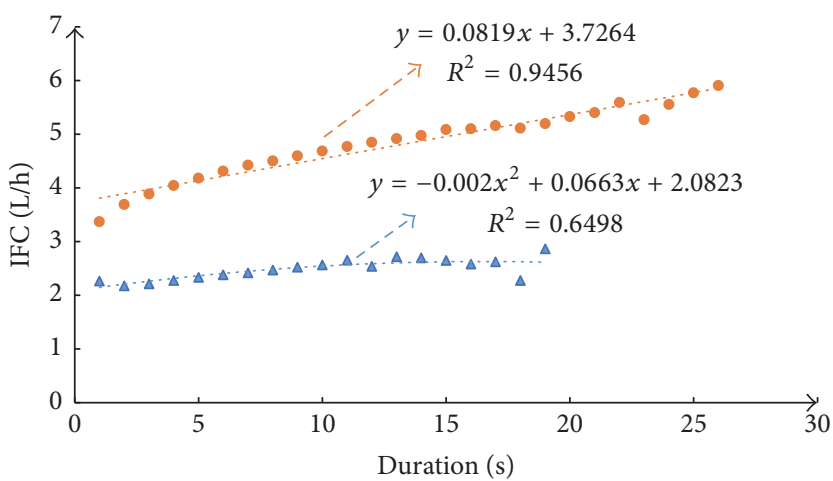

- Acceleration

$\triangle$ Cruising

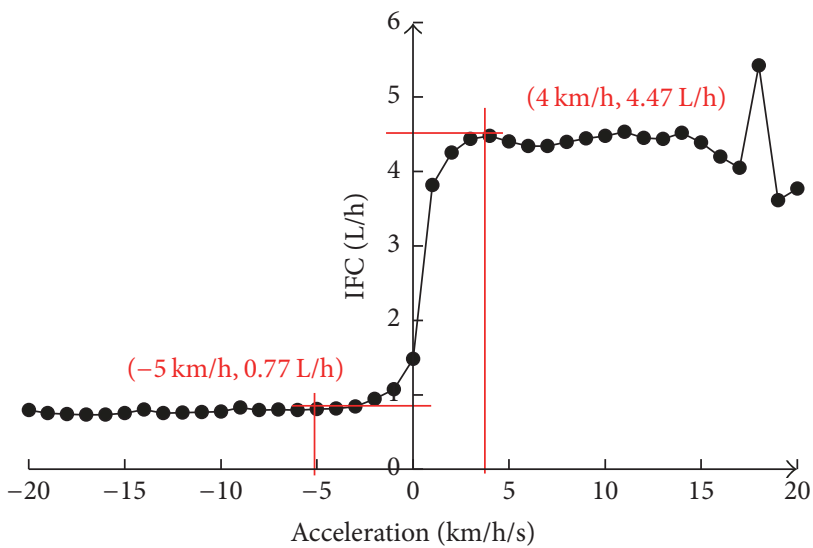

(b) Acceleration-IFC

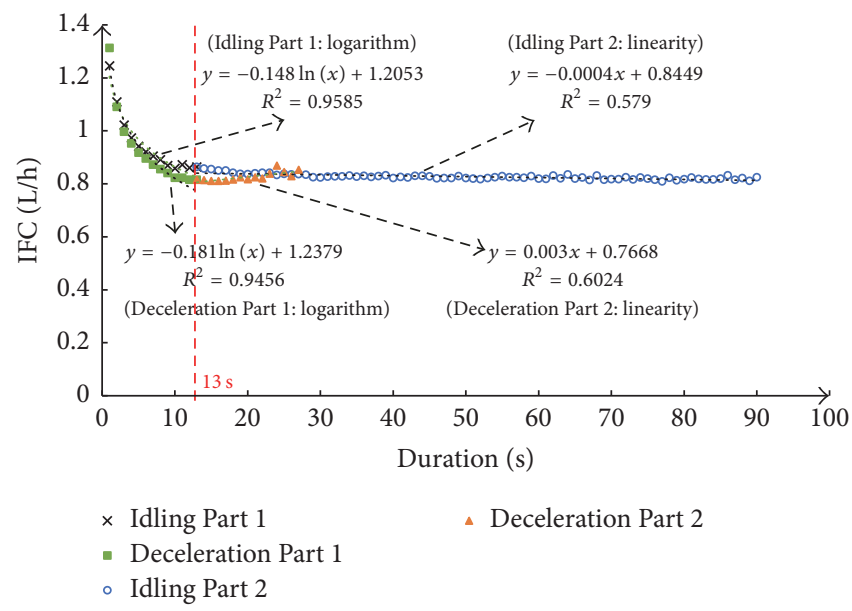

(d) Duration-IFC (deceleration and idling)

Figure 3: Influence of three parameters on vehicle fuel consumption. Note. Unit of acceleration is $\mathrm{km} / \mathrm{h} / \mathrm{s}$ instead of $\mathrm{m} / \mathrm{s}^{2}$. The reason is that the values using $\mathrm{km} / \mathrm{h} / \mathrm{s}$ as unit are in accordance with the ones displayed on the dashboard, which are easy to understand for drivers.

Figure 3(a) shows that FPH decreases rapidly when speed is lower than $30 \mathrm{~km} / \mathrm{h}$ and then tends to be stable with the increase of speed. Previous researches suggest that wind resistance increases sharply when speed is greater than $96 \mathrm{~km} / \mathrm{h}(60 \mathrm{mph})$ and consequently results in huge fuel consumption [23]. The data with speed greater than $100 \mathrm{~km} / \mathrm{h}$ are overlooked; therefore this situation is not included nor discussed in this research.

Figure 3(b) shows that IFC in acceleration is apparently higher than in deceleration. When accelerating, IFC first increases rapidly and then remains stable, which may be caused by the engine feature limitation. When decelerating, IFC decreases with the increase of deceleration. Specifically, IFC is maintained at about $0.77 \mathrm{~L} / \mathrm{h}$ when deceleration is greater than $5 \mathrm{~km} / \mathrm{h} / \mathrm{s}$, nearly the same as idling (seen in Figure 3(d)). To explain this phenomenon, it is probably that clutch pedal is completely depressed and the fuel is only used to maintain the engine operation at high deceleration. Although IFC shows a decrease in high deceleration, FPH may have a different tendency. Thus, in this paper sharply decelerating is also considered as poor driving behavior.
Figure 3(c) shows that, with the increase of driving mode duration, IFC of acceleration linearly increases, while IFC of cruising increases slowly.

Figure 3(d) plots the relationship between IFC and duration time of deceleration and idling. It is revealed that IFC of idling and deceleration has similar performances. To better describe the tendency, the curves of deceleration and idling are separated as two parts at the point $13 \mathrm{~s}$ : the logarithm curve (Part 1) and linearity line (Part 2). As can be seen, IFC of deceleration and idling firstly decreases with logarithm curve and then tends to be stable.

3.2. Propose and Define Driving Events. By reviewing ecodriving advices mentioned in previous researches [24], nine driving events that are closely related to vehicle fuel consumption and can be recognized simply by the combination of three parameters above (speed, acceleration, and driving mode duration) are proposed. The nine driving events include Accelerating Sharply (AS), Decelerating Sharply (DS), Long-Time Accelerating (LA), Long-Time Idling (LI), Running with Low Speed (LS), Cruising with Higher Speed 
(HS), Starting Moderately (SM), Frequently Stop and Start (SS), and Braking Moderately (BM).

Definitions of every driving events are list as follows. The determination of thresholds used in definitions is explained later.

(1) AS (Accelerating Sharply): $a(t)>4 \mathrm{~km} / \mathrm{h} / \mathrm{s}$. A period with continuous instantaneous ASs is identified as one AS event.

(2) DS (Decelerating Sharply): $a(t)<5 \mathrm{~km} / \mathrm{h} / \mathrm{s}$. A period with continuous instantaneous DS is identified as one DS event.

(3) LA (Long-Time Accelerating): $T_{a}>5 / s$. Accelerating that lasts more than $5 \mathrm{~s}$ is identified as one LA event.

(4) LI (Long-Time Idling): $T_{i}>60 / s$. Idling that lasts more than $60 \mathrm{~s}$ is identified as one LI event.

(5) LS (Running with Low Speed). Average speed during 60 seconds is no more than $23 \mathrm{~km} / \mathrm{h}$ (see (2)), and a period with continuous LSs is identified as one LS event.

$$
\text { ave }(s(t), s(t-1), \ldots, s(t-59)) \leq 23 \mathrm{~km} / \mathrm{h} \text {. }
$$

(6) HS (Cruising with Higher Speed). When driving, during 5 seconds, one has the following:

(i) Average speed is no less than $60 \mathrm{~km} / \mathrm{h}$; see (3)

(ii) Instantaneous acceleration is no more than $1 \mathrm{~km} / \mathrm{h}$; see (4)

(iii) Speed standard deviation is no more than $1.5 \mathrm{~km} / \mathrm{h}$; see (5)

(iv) Speed variation is no more than $1 \mathrm{~km} / \mathrm{h}$; see (6)

(v) A period with continuous HS is identified as one HS event.

$$
\begin{aligned}
& \operatorname{ave}(s(t), s(t-1), s(t-2), s(t-3), s(t-4)) \\
& \quad \geq 60 \mathrm{~km} / \mathrm{h} \\
& \max (a(t), a(t-1), a(t-2), a(t-3), a(t-4)) \\
& \quad \leq 1 \mathrm{~km} / \mathrm{h} / \mathrm{s} \\
& \operatorname{std}(s(t), s(t-1), s(t-2), s(t-3), s(t-4)) \\
& \quad \leq 1.5 \mathrm{~km} / \mathrm{h} \\
& \operatorname{abs}(s(t)-s(t-4)) \leq 1 \mathrm{~km} / \mathrm{h} .
\end{aligned}
$$

(7) SM (Starting Moderately). When vehicle accelerates from idling, during 5 seconds, one has the following:

(i) Speed variation $\in[10,20] \mathrm{km} / \mathrm{h} / \mathrm{s}$; see (7)

(ii) Product of driving mode codes equals 1 or 3; see (8)

(iii) Maximum acceleration is no more than $4 \mathrm{~km} / \mathrm{h} / \mathrm{s}$; see (9) (iv) A period with continuous SM is identified as one SM event.

$$
\begin{aligned}
& 10 \mathrm{~km} / \mathrm{h} \leq s(t)-s(t-4) \leq 20 \mathrm{~km} / \mathrm{h} \\
& m(t) \times m(t-1) \times m(t-2) \times m(t-3) \times m(t-4) \\
& \quad=1 \|=3 \\
& \max (a(t), a(t-1), a(t-2), a(t-3), a(t-4)) \\
& \quad \leq 4 \mathrm{~km} / \mathrm{h} / \mathrm{s} .
\end{aligned}
$$

(8) SS (Frequently Stop and Start). A vehicle idles within 3 seconds after starting from an idling. A period with continuous SS is identified as one SS event.

(9) BM (Braking Moderately). When decelerating, during 5 seconds, one has the following:

(i) Deceleration $\in[-25,-15] \mathrm{km} / \mathrm{h} / \mathrm{s}$; see (10)

(ii) Product of driving mode codes $\in(31,49)$; see (11)

(iii) Minimum acceleration is no less than $-5 \mathrm{~km} / \mathrm{h}$; see (12)

(iv) A period with continuous $\mathrm{BM}$ is identified one $\mathrm{BM}$ event.

$$
-25 \mathrm{~km} / \mathrm{h} / \mathrm{s} \leq s(t)-s(t-4) \leq-15 \mathrm{~km} / \mathrm{h} / \mathrm{s}
$$

31

$$
\begin{aligned}
< & m(t) \times m(t-1) \times m(t-2) \times m(t-3) \\
& \times m(t-4)<49 \\
\min (a(t), a(t-1), a(t-2), a(t-3), a(t-4)) & \\
\geq & -5 \mathrm{~km} / \mathrm{h},
\end{aligned}
$$

where $a(t)$ is acceleration on $t$ second, $\mathrm{km} / \mathrm{h} / \mathrm{s}, T_{a}$ is accelerating duration time, $s, T_{i}$ is decelerating duration time, $s, s(t)$ is speed on $t$ second, $\mathrm{km} / \mathrm{h}$, ave, std, abs, max, and min are calculating the sum, standard deviation, maximum value, and minimum value of given arguments, and $m(t)$ is driving mode code of $t$ second, 1, 2, 3, 4 index accelerating, decelerating, cruising, and idling, respectively.

\subsection{Threshold Determination}

3.3.1. Acceleration and Deceleration Threshold. Acceleration threshold is used to define AS, and it is also used in the definition of SM (see (7), (9)). Besides, deceleration threshold is used in definition of DS and BM (see (10), (12)). According to models shown in Figure 3(b), the rate of mutation of instantaneous fuel consumption (RMF) between two contiguous acceleration values is used as a measurement to determine the threshold. RMF of every acceleration value is calculated as (13) and then plotted in Figure 4.

$$
\mathrm{RMF}_{a}=\frac{g(a)-g(a-1)}{g(a-1)}, \quad a \in[1,20],
$$




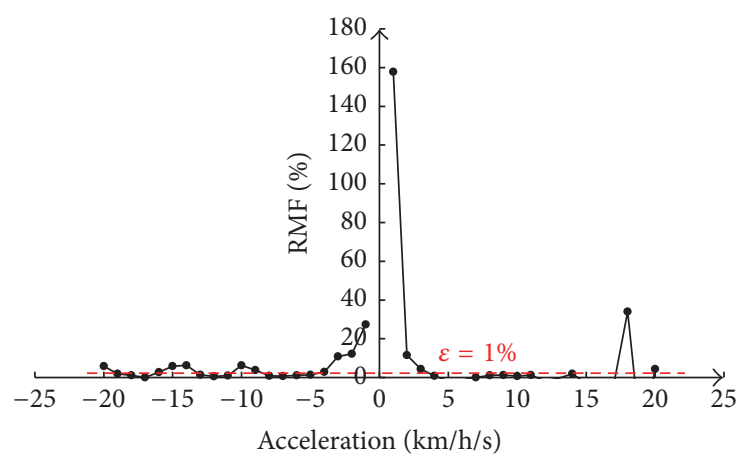

FIGURE 4: RMF of acceleration.

where $\mathrm{RMF}_{a}$ is RMF of given acceleration value and $g(a)$ is instantaneous fuel consumption when acceleration is $a$.

Figure 4 shows that as acceleration (deceleration) increases, RMF firstly decreases rapidly and then maintains stable. In terms of accelerating, IFC is thought of achieving maximum when RMF is less than a threshold $\varepsilon$. The corresponding acceleration value is determined as acceleration threshold because it is better not to accelerate with so big acceleration for its higher IFC. As for decelerating, IFC is thought of achieving minimum (nearly the same as idling) when RMF is less than a threshold $\varepsilon$. Obviously, drivers have not taken engine brake in this case. Taking $\varepsilon=1 \%$, acceleration and deceleration thresholds are $4 \mathrm{~km} / \mathrm{h} / \mathrm{s}$ and $-5 \mathrm{~km} / \mathrm{h} / \mathrm{s}$, respectively.

3.3.2. Low Speed Threshold and High Speed Threshold. Low speed threshold and high speed threshold are used in LS (see (2)) and HS (see (3)), respectively, to determine if a vehicle is in high-fuel-consumption state or in fuel economy state. Similarly with acceleration, RMF between two contiguous speed values is used in the determination of speed threshold. RMF of every speed values is calculated as (14) and then plotted in Figure 5.

$$
\mathrm{RMF}_{\mathrm{s}}=\frac{g(s)-g(s-1)}{g(s-1)}, \quad a \in[1,20],
$$

where $\mathrm{RMF}_{s}$ is RMF of given speed value and $g(s)$ is instantaneous fuel consumption when speed is $s$.

According to Figure 5, a vehicle is thought of getting rid of low speed state when RMF is less than a threshold $\varepsilon_{1}$ and running in a high speed state when RMF is less than a threshold $\varepsilon_{2}$. Taking $\varepsilon_{1}=3 \%$ and $\varepsilon_{2}=1 \%$, low speed threshold and high speed threshold are $23 \mathrm{~km} / \mathrm{h} / \mathrm{s}$ and $60 \mathrm{~km} / \mathrm{h} /$ s, respectively.

3.3.3. Time Threshold in LA. The period of $5 \mathrm{~s}$ is long enough for drivers to accelerate to a higher speed (if accelerating with $4 \mathrm{~km} / \mathrm{h} / \mathrm{s}$, speed increases $20 \mathrm{~km} / \mathrm{h}$ ). Meanwhile, operation complexity of laddering acceleration every $5 \mathrm{~s}$ is acceptable for driver.

3.3.4. Time Threshold in LS and LI. Considering that both stopping time at intersections and passengers getting on/off time in Beijing are generally less than $60 \mathrm{~s}$, thresholds used in LS and LI are set to $60 \mathrm{~s}$ to avoid misjudging these two cases. Moreover, in terms of LI, Union of Concerned Scientists [25] also suggests that idling time is better not beyond $60 \mathrm{~s}$.

3.3.5. Time Threshold in HS, SM, and BM. For HS, it seems difficult for drivers to cruise with stable speed. For SM and $\mathrm{BM}$, the period of $5 \mathrm{~s}$ is long enough for drivers to accelerate/decelerate to a higher/lower speed (if accelerating/decelerating with $4 /-5 \mathrm{~km} / \mathrm{h} / \mathrm{s}$, speed increases/decreases by $20 / 25 \mathrm{~km} / \mathrm{h}$ ).

3.3.6. Product of Driving Mode Codes Threshold SM and BM. Ranges of product of driving mode codes used in SM (see (8)) and BM (see (11)) are calculated to ensure that the whole driving events process is an acceleration or deceleration process (allowing temporal cruising). Enumeration method is used to determine the range, as shown in Table 1.

3.3.7. Speed Standard Deviation Threshold in HS. Speed standard deviation used in LS (see (5)) is used to ensure that vehicle speed oscillation is less than $1 \mathrm{~km} / \mathrm{h}$. Similar to product of driving mode codes, threshold 1.5 of speed standard deviation is determined by enumeration method as well.

\section{Eco-Driving Behavior Evaluation Modeling and Advices Providing}

307 drivers were randomly divided into five groups. Four of the five groups $(61 * 4=244$ samples) were used to establish the model (training set). The remaining group (63 samples) was used to verify the model (test set). As preparations for modeling, two tasks were completed at first: (1) identifications of nine driving events of every samples and (2) fuel-related hundred-mark system establishment.

4.1. Nine Driving Events Identification of Every Sample and Description. According to the definition listed above, driving events were identified and the average frequency every $50 \mathrm{~km}$ of nine driving events was calculated to normalize the travel distance. In the data collection day, average frequency of driving events every $50 \mathrm{~km}$ was shown in Table 2. Meanwhile, average frequency of driving events every $1 \mathrm{~km}$ was also shown to have a better view. 


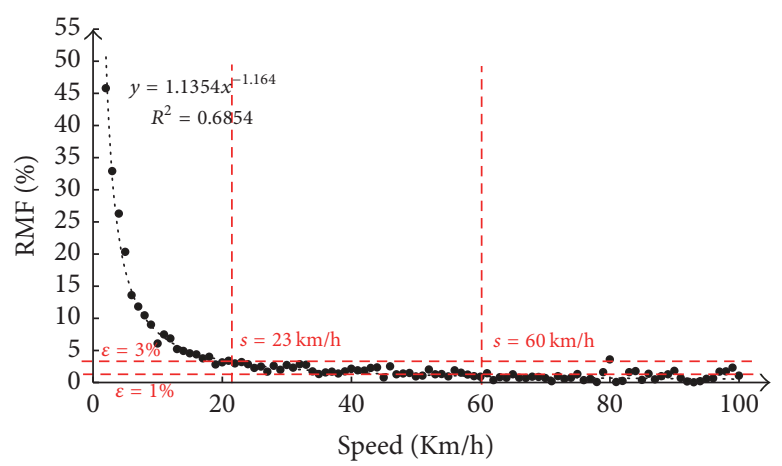

Figure 5: RMF of speed.

TABLE 1: Enumeration of the product of driving mode codes in SM and BM.

\begin{tabular}{|c|c|c|c|c|c|c|c|}
\hline \multirow{2}{*}{ Cases } & \multicolumn{5}{|c|}{ Driving mode code on every second } & \multirow{2}{*}{ Product } & \multirow{2}{*}{ Remarks } \\
\hline & $1 \mathrm{~s}$ & $2 \mathrm{~s}$ & $3 \mathrm{~s}$ & $4 \mathrm{~s}$ & $5 \mathrm{~s}$ & & \\
\hline \multicolumn{8}{|c|}{ SM } \\
\hline Case 1 & 1 & 1 & 1 & 1 & 1 & 1 & Accelerating continuously \\
\hline Case 2 & 1 & 1 & 1 & 1 & 2 & 2 & Having decelerating \\
\hline Case 3 & 1 & 1 & 1 & 1 & 3 & 3 & Accelerating with temporal cruising \\
\hline Case 4 & 1 & 1 & 1 & 1 & 4 & 4 & Having idling \\
\hline Case 5 & 1 & 1 & 1 & 3 & 3 & 9 & Too much cruising \\
\hline \multicolumn{8}{|c|}{$\mathrm{BM}$} \\
\hline Case 1 & 2 & 2 & 2 & 2 & 1 & 16 & Having accelerating \\
\hline Case 2 & 2 & 2 & 2 & 2 & 2 & 32 & Decelerating continuously \\
\hline Case 3 & 2 & 2 & 2 & 2 & 3 & 48 & Decelerating with temporal cruising \\
\hline Case 4 & 2 & 2 & 2 & 3 & 3 & 72 & Too much cruising \\
\hline
\end{tabular}

Note. (1) Italicized cases stand for the cases that could be identified as an SM process or BM process; (2) the order of driving mode codes is not necessarily ranked as list in table. For example, 2-2-3-2-2 is also identified as case $3(\mathrm{BM}) ;(3)$ driving mode code 1, 2, 3, 4 index accelerating, decelerating, cruising, and idling, respectively.

TABLE 2: Average frequency of driving events every $50 \mathrm{~km}$ and $1 \mathrm{~km}$.

\begin{tabular}{lccccccccc}
\hline Driving events & AS & DS & LA & LI & LS & HS & SM & SS & BM \\
\hline Frequency $/ 50 \mathrm{~km}$ & 122.4 & 88.8 & 136.4 & 8.1 & 28.1 & 34.0 & $53.8 \%$ & 1.2 & $32.9 \%$ \\
Frequency $/ \mathrm{km}$ & 2.44 & 1.77 & 2.72 & 0.16 & 0.56 & 0.68 & $1.08 \%$ & 0.02 & $0.65 \%$ \\
\hline
\end{tabular}

It should be noted that unit of SM and BM was the ratio of SM/All-Staring and BM/All-Braking instead of the frequency of SM and BM. The reason was that the more the SM or $\mathrm{BM}$ occurred, the better the fuel economy in general is. But in fact, more SM and BM were always accompanied with more starting and braking processes, which would increase fuel consumption compared with cruising. Thus, the ratio of SM/All-Staring and BM/All-Braking might be better as indicators.

Table 2 indicates that during a certain travel distance, drivers are prone to AS and LA (nearly 2.5 times per kilometer), while being less to SS and LI. LS occurs about every $2 \mathrm{~km}$ on average, which may be caused by the close distance between intersections and frequent traffic jams in Beijing.
4.2. Fuel-Related Hundred-Mark System. The evaluation of driver's eco-driving behavior was aimed at helping drivers to promote eco-driving behavior. Hence, a simple and easy-tounderstand indicator was needed. In this paper, a hundredmark system was used to measure driver's eco-driving behavior. Scores of every driver was calculated by the following linear equation:

$$
\begin{aligned}
\operatorname{SCORE}_{a}=40+\left(1-\frac{(\mathrm{FPH}-6)}{14-6}\right) \times 60, & \\
& \mathrm{FPH} \in[6,14],
\end{aligned}
$$

where $\mathrm{SCORE}_{a}$ is scores of every driver and FPH is FPH of every driver, and it is calculated using (1). 
TABLE 3: Correlation between arguments.

\begin{tabular}{lccccccccc}
\hline Driving events & 1 & 2 & 3 & 4 & 5 & 6 & 7 & 8 \\
\hline$(1)$ & 1 & $.905^{* *}$ & $.680^{* *}$ & $.607^{* *}$ & $.769^{* *}$ & $-.566^{* *}$ & -.010 & $.271^{* *}$ & $.410^{* *}$ \\
$(2)$ & $.905^{* *}$ & 1 & $.747^{* *}$ & $.586^{* *}$ & $.745^{* *}$ & $-.550^{* *}$ & .007 & $.315^{* *}$ & $.565^{* *}$ \\
$(3)$ & $.680^{* *}$ & $.747^{* *}$ & 1 & $.684^{* *}$ & $.854^{* *}$ & $-.736^{* *}$ & .040 & $.312^{* *}$ & $.238^{* *}$ \\
$(4)$ & $.607^{* *}$ & $.586^{* *}$ & $.684^{* *}$ & 1 & $.787^{* *}$ & $-.613^{* *}$ & .013 & $.366^{* *}$ & .037 \\
$(5)$ & $.769^{* *}$ & $.745^{* *}$ & $.854^{* *}$ & $.787^{* *}$ & 1 & $-.775^{* *}$ & .009 & $.326^{* *}$ & $.191^{* *}$ \\
$(6)$ & $-.566^{* *}$ & $-.550^{* *}$ & $-.736^{* *}$ & $-.613^{* *}$ & $-.775^{* *}$ & 1 & .006 & $-.279^{* *}$ & .018 \\
$(7)$ & -.010 & .007 & .040 & .013 & .009 & .006 & 1 & $-.198^{* *}$ & $.199^{* *}$ \\
$(8)$ & $.271^{* *}$ & $.315^{* *}$ & $.312^{* *}$ & $.366^{* *}$ & $.326^{* *}$ & $-.279^{* *}$ & $-.198^{* *}$ & 1 \\
$(9)$ & $.410^{* *}$ & $.565^{* *}$ & $.238^{* *}$ & .037 & $.191^{* *}$ & .018 & $.199^{* *}$ & .002 \\
\hline
\end{tabular}

Note. (a) ** Correlation is significant at the 0.01 level; (b) (1)-(9) represent AS, DS, LA, LI, LS, HS, SM, SS, and BM, respectively.

TABLE 4: Results of PCA.

\begin{tabular}{lccc}
\hline Component & Total & \% of variance & Cumulative\% \\
\hline F1 & 4.793 & 53.258 & 53.258 \\
F2 & 1.377 & 15.302 & 68.560 \\
F3 & 1.059 & 11.763 & 80.324 \\
F4 & .715 & 7.940 & 88.264 \\
F5 & .391 & 4.349 & 92.613 \\
F7 & .303 & 3.369 & 95.982 \\
F8 & .190 & 2.110 & 98.092 \\
F9 & .118 & 1.310 & 99.402 \\
\hline
\end{tabular}

Obviously, the higher the fuel consumption, the lower the scores. Driver's minimum score was 40 and maximum was 100 corresponding to the maximum fuel consumption of $14 \mathrm{~L} / 100 \mathrm{~km}$ and the minimum of $6 \mathrm{~L} / 100 \mathrm{~km}$. When fuel consumption was lower than $6 \mathrm{~L} / 100 \mathrm{~km}$, score was 100 . When fuel consumption was higher than $14 \mathrm{~L} / 100 \mathrm{~km}$, score was 40 .

\subsection{Establishment of Eco-Driving Behavior Evaluation Model.}

Taking numbers of every driving events occurred during a certain distance as independent variables and vehicle fuel consumption as dependent variable, driver's eco-driving behavior evaluation model was established. Multiple linear regression was chosen as modeling method due to the fact that change of the arguments (i.e., the change of driver behavior to better or worse) could directly influence evaluation score. In this case, the reason why driver had high fuel cost could be intuitively provided, as well as advices that which vehicle manipulation should be improved or eliminated.

When modeling, strong collinearity between arguments was observed, as shown in Table 3.

In this case, directly using linear regression might cause model distortion. Therefore, principal component analysis (PCA) was used to eliminate collinearity.

First, data of 244 samples were normalized using $Z$ standardization, as follows:

$$
x^{*}=\frac{x-\mu}{\sigma},
$$

where $x^{*}$ is normalized value, $x$ is raw value, $\mu$ is mean, and $\sigma$ is standard deviation.
Second, using PCA, the result was shown in Table 4.

The first three principal components were extracted since their Eigen values were greater than 1. The expressions of every principal components were listed as follows:

$$
\begin{aligned}
F_{1}= & 0.181 N_{\mathrm{AS}}+0.184 N_{\mathrm{DS}}+0.187 N_{\mathrm{LA}}+0.167 N_{\mathrm{LI}} \\
& +0.195 N_{\mathrm{LS}}-0.172 N_{\mathrm{HS}}-0.01 N_{\mathrm{SM}} \\
& +0.088 N_{\mathrm{SS}}+0.06 N_{\mathrm{BM}} \\
F_{2}= & 0.155 N_{\mathrm{AS}}+0.227 N_{\mathrm{DS}}-0.038 N_{\mathrm{LA}}-0.175 N_{\mathrm{LI}} \\
& -0.068 N_{\mathrm{LS}}+0.138 N_{\mathrm{HS}}+0.375 N_{\mathrm{SM}} \\
& -0.322 N_{\mathrm{SS}}+0.592 N_{\mathrm{BM}} \\
F_{3}= & 0.145 N_{\mathrm{AS}}+0.217 N_{\mathrm{DS}}-0.129 N_{\mathrm{LA}}-0.183 N_{\mathrm{LI}} \\
& -0.136 N_{\mathrm{LS}}+0.270 N_{\mathrm{HS}}-0.681 N_{\mathrm{SM}} \\
& +0.367 N_{\mathrm{SS}}+0.369 N_{\mathrm{BM}},
\end{aligned}
$$

where $F_{1}$ is the first principal component, $F_{2}$ is the second principal component, $F_{3}$ is the third principal component, $N_{\text {AS }}$ is number of AS occurring every $50 \mathrm{~km}, N_{\mathrm{DS}}$ is number of DS occurring every $50 \mathrm{~km}, N_{\mathrm{LA}}$ is number of LA occurring every $50 \mathrm{~km}, N_{\mathrm{LI}}$ is number of LI occurring every $50 \mathrm{~km}, N_{\mathrm{LS}}$ is number of LS occurring every $50 \mathrm{~km}, N_{\mathrm{HS}}$ is number of HS occurring every $50 \mathrm{~km}, N_{\mathrm{SM}}$ is number of SM occurring every $50 \mathrm{~km}, N_{\mathrm{SS}}$ is number of SS occurring every $50 \mathrm{~km}$, and $N_{\mathrm{BM}}$ is number of BM occurring every $50 \mathrm{~km}$.

Third, taking three principal components as arguments and vehicle fuel consumption as depend variable, multiple 
TABLE 5: Influence on score when driving event occurs once (every $50 \mathrm{~km}$ ).

\begin{tabular}{lccccccccc}
\hline Driving events & AS & DS & LA & LI & LS & HS & SM & SS \\
\hline Coefficients & -0.03 & -0.04 & -0.05 & -0.44 & -0.18 & 0.07 & 0.02 & -1.05 & 0.11 \\
Rank & 8 & 7 & 6 & 2 & 3 & 5 & 9 & 1 & 4 \\
\hline
\end{tabular}

linear regression was used to establish driver's eco-driving behavior evaluation model. The model was expressed as follows:

$$
\begin{aligned}
\operatorname{SCORE}_{M} & =80.549-6.927 F_{1}+1.455 F_{2}+0.565 F_{3}, \\
R^{2} & =0.823,
\end{aligned}
$$

where $\mathrm{SCORE}_{M}$ is model score of every driver.

In this model, the relationship between the three principal components and score was presented. Meanwhile, the principal components are the linear combination of nine arguments (i.e., the frequency of nine driving events occurs every $50 \mathrm{~km}$ ). Thereby the score could be expressed by nine arguments also with linear form. Coefficients of every argument were shown in Table 5. The coefficients could also indicate how score will change when driver performs one driving event.

Table 5 suggests that, in every $50 \mathrm{~km}$, once SS and LI occur, scores decrease by 1.05 and 0.44 , respectively, which indicates that the occurrences of these two driving events will result in obvious increase of fuel consumption. Thus, drivers should pay more attention to avoid SS and LI to achieve higher fuel efficiency.

4.4. Model Validation. The remaining group (63 samples) was used to verify the eco-driving behavior evaluation model. Vehicle fuel consumption during one day was calculated using vehicle operation data and then converted to scores. Nine driving events were also identified and their frequencies (every $50 \mathrm{~km}$ ) were counted. Substituting these nine arguments into the evaluation model, model scores were obtained.

Relative error was used to measure the model accuracy.

$$
E_{i}=\frac{\left|\operatorname{SCORE}_{M}-\mathrm{SCORE}_{A}\right|}{\mathrm{SCORE}_{A}}
$$

$E_{i}$ indicates the relative error between fuel consumptioncalculating score and model score of $i$ th driver. Taking average relative error as measurement, validation results showed that model error was $3.28 \%$; that is, model accuracy was 96.72\%.

Relationship between fuel consumption-calculating score and model score was plotted in Figure 6.

4.5. Driving Advices. Based on the definition of the nine driving events, nine eco-driving advices described as several processes were proposed as follows.

(1) AS. Avoid sharp acceleration. When accelerating, it is better to accelerate to $15 \mathrm{~km} / \mathrm{h}$ within $4 \mathrm{~s}$.
(2) DS. Avoid sharp deceleration. When decelerating, it is better to decelerate to $20 \mathrm{~km} / \mathrm{h}$ with $4 \mathrm{~s}$.

(3) LA. Avoid long-time acceleration. Take laddering accelerating operation to have vehicle instantaneously cruising or decelerating within accelerating process. It is better not to continuously accelerate beyond $5 \mathrm{~s}$.

(4) LI. Avoid long-time idling. Anticipating traffic condition and signal phase in intersection, drivers should switch off engine when predicting stopping more than $60 \mathrm{~s}$.

(5) LS. Make travel planning and try to bypass jam roads.

(6) HS. Try to maintain higher speed but not over speed.

(7) SM. When starting, it is better to accelerate to $20 \mathrm{~km} / \mathrm{h}$ within $5 \mathrm{~s}$.

(8) SS. Under congestion condition, it is better to start when the space from the front car is more than half a car to avoid frequently stopping and starting.

(9) $B M$. When decelerating, it is better to decelerate to $15-20 \mathrm{~km} / \mathrm{h}$ within $5 \mathrm{~s}$.

\section{Discussion}

In our research, the database used in the definition of driving events and establishment of evaluation model is collected in only one day. It seems that the dataset might not be large enough, but the longer average operation time of taxicabs (10.3 hours) is able to cover various traffic conditions and road types.

In some previous studies, external circumstances such as road slope, weather condition, traffic condition, and vehicle load have influences on car's fuel consumption. However, in this paper, these factors are not taken into consideration for the following reasons:

(1) The information is difficult to collect and the integration between different data sources is complex. Thus, the application of paper results may be limited.

(2) The general improvement of driver' manipulation skills is mainly focused on, no matter how the external conditions change. For example, the consideration of traffic condition before travels can help drivers to avoid traffic congestions and yield a lower energy usage. Nevertheless, if drivers engage in slow traffic flow, eco-driving manipulation still can save fuel compared with normal driving. Similarly, weather condition can influence vehicle fuel consumption, while the overall performance of driving manipulation for 


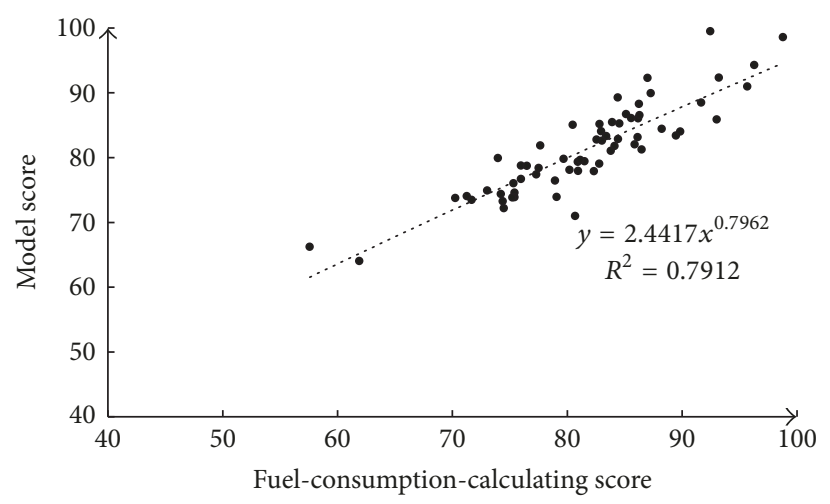

FIGURE 6: Relationship between fuel consumption-calculating score and model score.

specific driver is steady. In other words, if vehicle operating parameters (speed, acceleration, and driving mode duration), which can be regarded as the results of driver manipulation, are in line with eco-driving advices, fuel consumption will be lower than that when driving normally under the same external circumstance.

In Section 2, data with vehicle speed more than $100 \mathrm{~km} / \mathrm{h}$ (illegal drive) are ignored. One may doubt that this treatment may influence modeling. However, this paper mainly deals with the eco-driving under normal driving conditions. Moreover, data that speed is more than $100 \mathrm{~km} / \mathrm{h}$ only occupy $1 \%$ in overall database. Thus the ignorance is irrelevant for the modeling and result.

According to the model coefficients, it appears that AS, DS, LA, LI, LS, and SS (bad manipulations) will result in high fuel consumption for their negative coefficients in the model expression. On the contrary, HS, SM, and BM (good manipulations) will improve the evaluation scores for their positive coefficients in evaluation model. This phenomenon is in accordance with previous literature and common sense, which verifies the methodology of this paper from other sides.

In Section 3, consecutive same driving events are regarded as one driving event; that is, the frequency is taken into the model but the duration time of every event is ignored. This is because of the fact that the goal of this research is to inform drivers that how many times they take bad/good manipulations, and then they are expected to avoid/take more these manipulations. In other words, numbers of these events are advices to decrease instead of the duration time of every event.

The limitation of this research is about the thresholds used when defining driving events. According to data observation and driving experience, a series of parameter thresholds used in evaluation model are proposed and they work well in the modeling process. Nevertheless, these thresholds could not be assured to be optimal. On the other hand, the robustness of this model was not fully analyzed for the data limitation. But as a method for eco-driving behavior evaluation, the model could be applied to the practice after calibration and optimization with new data.

The foundation of this research is based on the taxicabs' operation data. It will be beneficial to promote these research findings to other types of vehicles. What is more, the nine driving events could be identified by only speed parameter. So when practically applying, cellphone could be used as both data source and feedback media after further validation.

Furthermore, driving-events-based evaluation model could only help drivers improve their driving behavior in the nine aspects. In future work, machine learning could be introduced into the evaluation modeling to explore hidden factors that influence fuel consumption and evaluate driver's eco-driving behavior more accurately. A fuel consumption model that fits the fuel consumption characteristics of Chinese vehicles will be developed too.

\section{Conclusion}

This paper establishes a driver eco-driving behavior evaluation model based on vehicle operating and fuel consumption data. First, the influence of three vehicle operating parameters including speed, acceleration, and driving mode duration on fuel consumption is analyzed. Then, nine driving events that are fuel consumption related are proposed. Using PCA and multiple linear regression, an eco-driving behavior evaluation model is established and nine corresponding eco-driving advices are provided.

The establishment of this model provides a quantitative and intuitive way to evaluate the effect of drivers' behavior on fuel consumption and several procedural-driving-eventsbased eco-driving advices. The outcomes of this paper could be applied directly to the development of eco-driving support device for its simple but accurate algorithms, laying the foundation for the eco-driving promotion in China.

\section{Notation}

FPH: Fuel consumption per hundred kilometers $(\mathrm{L} / 100 \mathrm{~km})$

IFC: Instantaneous fuel consumption $(\mathrm{L} / \mathrm{h})$

AS: Accelerating Sharply (driving event)

DS: Decelerating Sharply (driving event)

LA: Long-Time Accelerating (driving event)

LI: Long-Time Idling (driving event)

LS: Running with Low Speed (driving event)

HS: Cruising with Higher Speed (driving event) 
SM: Starting Moderately (driving event)

SS: Frequently Stop and Start (driving event)

BM: Braking Moderately (driving event)

RMF: Mutation of instantaneous fuel consumption (\%)

PCA: Principal component analysis (algorithm).

\section{Conflicts of Interest}

The authors declare that they have no conflicts of interest.

\section{Acknowledgments}

The authors would like to acknowledge the project of National Natural Science Foundation of China no. 61672067 for supporting this research.

\section{References}

[1] China Statistical Yearbook, "National Bureau of Statistics of the People's Republic of China," 2016, http://www.stats.gov.cn/tjsj/ ndsj/2015/indexch.htm.

[2] Y. B. Cui, "Study of automobile emissions saving countermeasures," Science \& Technology Vision, vol. 4, p. 190, 2014.

[3] I. De Vlieger, "On-board emission and fuel consumption measurement campaign on petrol-driven passenger cars," Atmospheric Environment, vol. 31, no. 22, pp. 3753-3761, 1997.

[4] J. Van Mierlo, G. Maggetto, E. Van De Burgwal, and R. Gense, "Driving style and traffic measures - Influence on vehicle emissions and fuel consumption," Proceedings of the Institution of Mechanical Engineers, Part D: Journal of Automobile Engineering, vol. 218, no. 1, pp. 43-50, 2004.

[5] Pham and D. Nam et al., Fueoogle: a participatory sensing fuelefficient maps application, 2009.

[6] M. Barth, K. Boriboonsomsin, and A. Vu, "EnvironmentallyFriendly navigation," in Proceedings of the 10th International IEEE Conference on Intelligent Transportation Systems, ITSC 2007, pp. 684-689, October 2007.

[7] K. Boriboonsomsin, M. J. Barth, W. Zhu, and A. Vu, "Ecorouting navigation system based on multisource historical and real-time traffic information," IEEE Transactions on Intelligent Transportation Systems, vol. 13, no. 4, pp. 1694-1704, 2012.

[8] E. Yao and Y. Song, "Study on eco-route planning algorithm and environmental impact assessment," Journal of Intelligent Transportation Systems: Technology, Planning, and Operations, vol. 17, no. 1, pp. 42-53, 2013.

[9] M. Barth, S. Mandava, K. Boriboonsomsin, and H. Xia, "Dynamic ECO-driving for arterial corridors," in Proceedings of the IEEE Forum on Integrated and Sustainable Transportation System (FISTS '11), pp. 182-188, IEEE, Vienna, Austria, July 2011.

[10] S. Mandava, K. Boriboonsomsin, and M. Barth, "Arterial velocity planning based on traffic signal information under light traffic conditions. intelligent transportation systems," in Proceedings of the 2009 12th International IEEE Conference on Intelligent Transportation Systems, pp. 160-165, October 2009.

[11] Z. Chen, An Optimization Model for Eco-Driving at Signalized Intersection, AM University, 2013.

[12] K. Boriboonsomsin, A. Vu, and M. Barth, Eco-driving: pilot evaluation of driving behavior changes among us drivers, University of California Transportation Center, 2010.
[13] R. Frank, G. Castignani, R. Schmitz, and T. Engel, "A novel ecodriving application to reduce energy consumption of electric vehicles," in Proceedings of the 2013 2nd IEEE International Conference on Connected Vehicles and Expo, ICCVE 2013, pp. 283-288, IEEE, December 2013.

[14] R. Araújo, Â. Igreja, R. De Castro, and R. E. Araújo, “Driving coach: a smartphone application to evaluate driving efficient patterns," in Proceedings of the 2012 IEEE Intelligent Vehicles Symposium, IV 2012, pp. 1005-1010, IEEE, Alcala de Henares, Spain, June 2012.

[15] Y. Wu, X. Zhao, J. Rong, Y. Liu, and L. Xu, "Potential of Ecodriving in Reducing Fuel Consumption and Emissions Based on a Driving Simulator," Journal of Beijing University of Technology, vol. 41, pp. 1211-1218, 2015.

[16] Y. Wu, X. Zhao, C. Chen, J. Rong, and Y. Zhang, "Development of an internet + based eco-driving support platform and its application: a case study in beijing taxicabs," Transportation Research Board 17-05681, 2017.

[17] J. C. Ferreira, J. De Almeida, and A. R. Da Silva, "The impact of driving styles on fuel consumption: a data-warehouse-anddata-mining-based discovery process," IEEE Transactions on Intelligent Transportation Systems, vol. 16, no. 5, pp. 2653-2662, 2015.

[18] D. Vangi and A. Virga, "Evaluation of energy-saving driving styles for bus drivers," Proceedings of the Institution of Mechanical Engineers, Part D: Journal of Automobile Engineering, vol. 217, no. 4, pp. 299-305, 2003.

[19] G. Kedar-Dongarkar and D. Manohar, "Driver classification for optimization of energy usage in a vehicle," Procedia Computer Science, vol. 8, pp. 388-393, 2012.

[20] Z. Yunming, Experimental and Simulation Investigation and Operating Characteristic of a Hybrid Hydraulic Passenger Car with an Output Coupled Power-Split Transmission, University of Technology, Beijing, China, 2014.

[21] GB 7528-2012, "Safety specifications for power-driven vehicles operating on roads".

[22] K. Ahn, H. Rakha, A. Trani, and M. van Aerde, "Estimating vehicle fuel consumption and emissions based on instantaneous speed and acceleration levels," Journal of Transportation Engineering, vol. 128, no. 2, pp. 182-190, 2002.

[23] M. Barth and K. Boriboonsomsin, "Traffic congestion and greenhouse gases," ACCESS Magazine, vol. 1, 2009.

[24] K. Jakobsen, S. C. H. Mouritsen, and K. Torp, "Evaluating ecodriving advice using GPS/CANBus data," in Proceedings of the 21st ACM SIGSPATIAL International Conference on Advances in Geographic Information Systems, ACM SIGSPATIAL GIS 2013, pp. 44-53, November 2013.

[25] Union of Concerned Scientists, "How to Maximize Your Vehicle's Fuel Economy," 2016, http://www.ucsusa.org/cleanvehicles/fuel-efficiency/how-to-maximize-fuel-economy\#.VuEQ8_ 19671. 


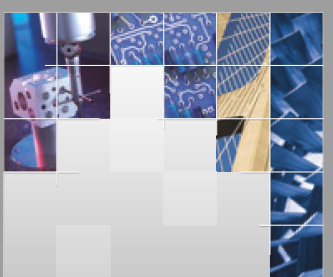

\section{Enfincering}
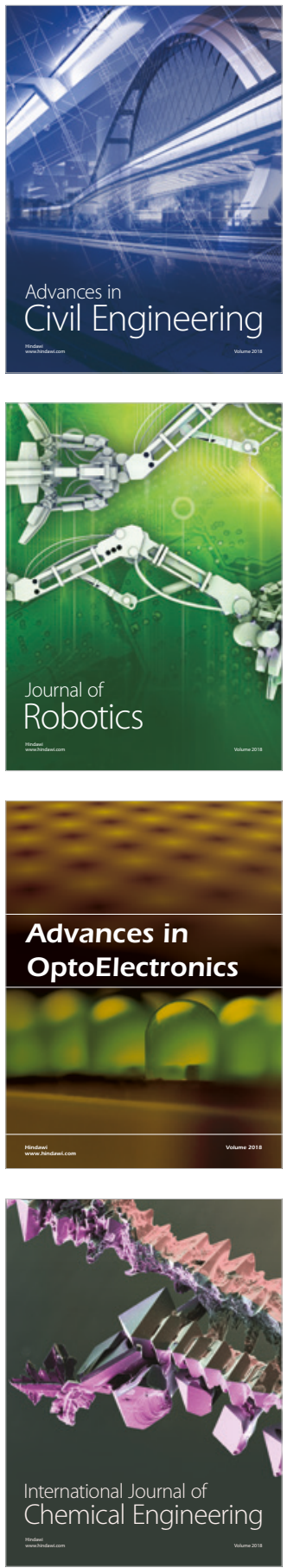

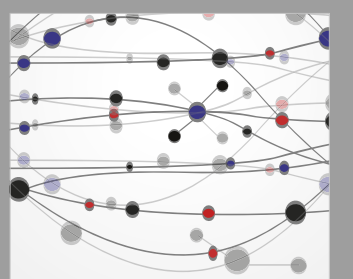

\section{Rotating \\ Machinery}

The Scientific World Journal

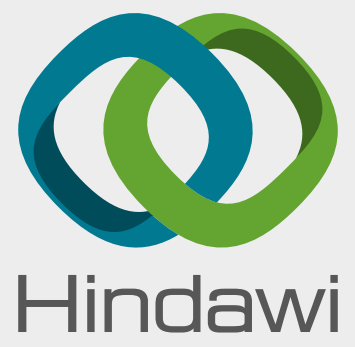

Submit your manuscripts at

www.hindawi.com
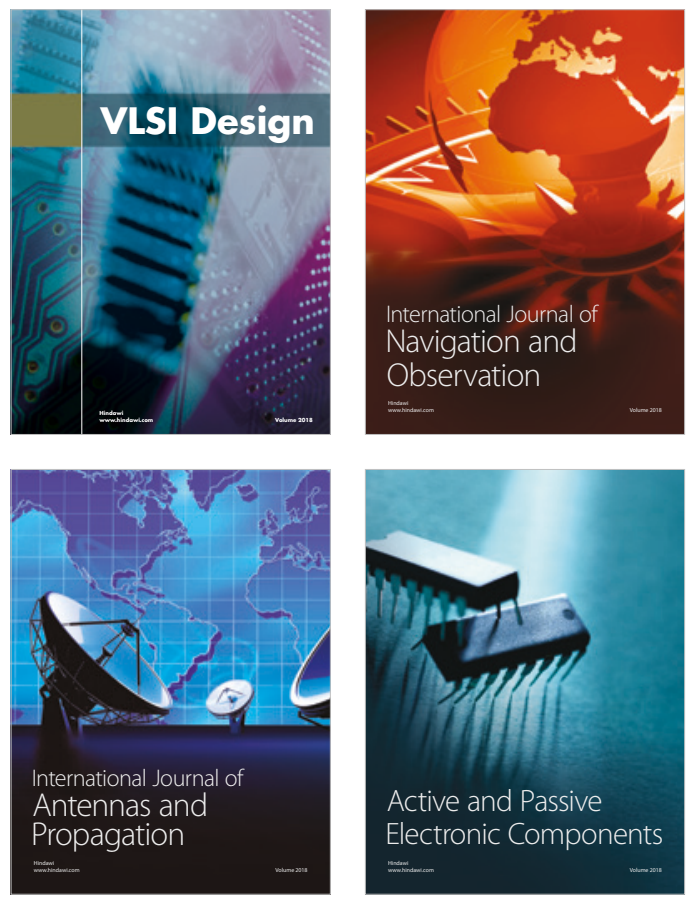
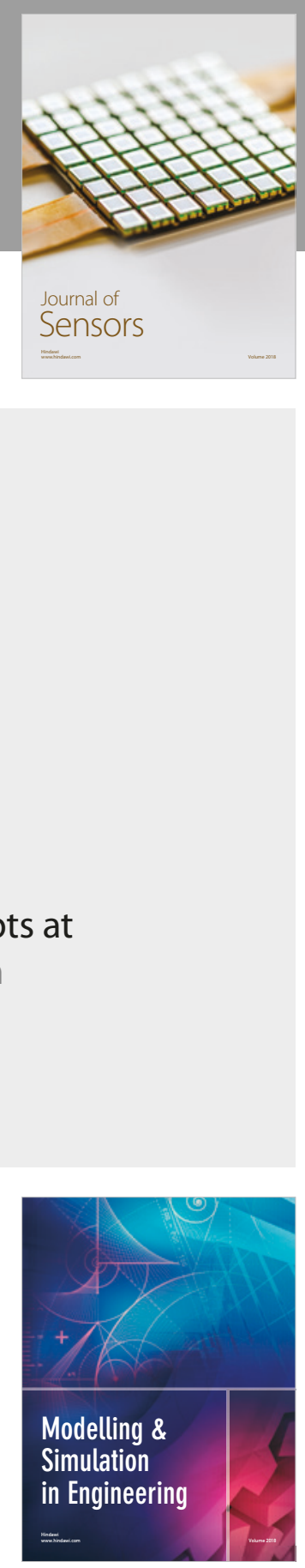

\section{Advances \\ Multimedia}
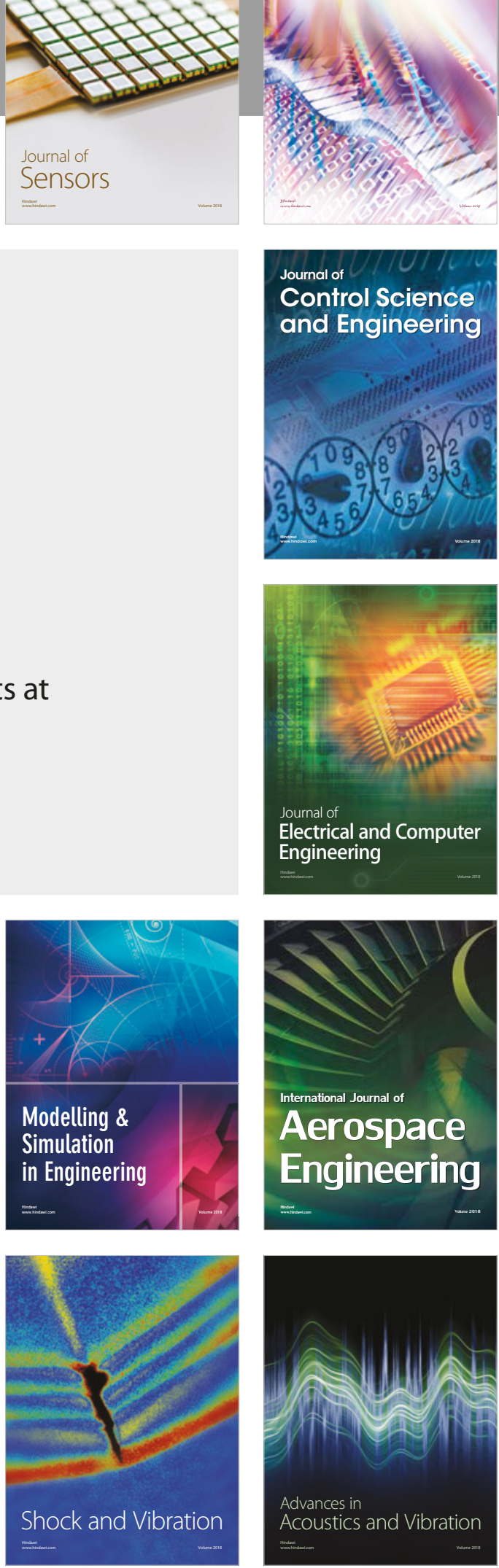